\title{
On fitting position of inlet and outlet pipes to elliptic cylindrical muffler chambers
}

\author{
Nguyen Manh Cuong* and Josuke Okda** \\ * Kumamoto Institute of Technology University, 4-22-1, Ikeda, Kumamoto, 860 Japan \\ **Faculty of Engineering, Kumamoto University, 2-39-1, Kurokami, Kumamoto, 860 Japan
}

(Received 30 June 1980)

\begin{abstract}
In order to make clear the characteristics of elliptic cylindrical chambers of a muffler, the distribution of sound pressure is calculated by solving the wave equations, assuming that the loss can be neglected. In addition, the effective frequency range is defined by theoretical investigation and verified by experiment on the formation of higher modes and on the position of input and output pipes.
\end{abstract}

PACS number 43 50.Gf

\section{INTRODUCTION}

There are two sorts of waves inside the cylindrical chamber of a muffler: the standing wave that propagates in the axial direction and the traverse wave that propagates in the radial direction. The latter occurs in the high frequency range. Regarding the circular cylindrical chamber, the noise will have a tendency to increase in this frequency range, far from decreasing, depending on the resonances of the higher order modes. ${ }^{1,2)}$

At present, in the silencing systems for exhaust noise of automobiles, the elliptic cylindrical chamber of mufflers are generally used, its characteristics however have not yet been throughly investigated theoretically.

In this paper, to make clear the characteristics of elliptical chamber, the theoretical calculations of the sound pressure inside the chamber are carried out by solving the wave equation with the assumption that the loss at the wall of a chamber can be neglected. The results are compared with findings from our experiment.

Additionally, in order to make wider the effective frequency range, the setting of input and output pipes are determined by an investigation of the shapes of higher modes formed inside the chamber.

\section{THEORY}

2.1 Solution of the Wave Equation

The general solution of the wave equation in elliptical co-ordinates is a combination of some functions as follows:

$$
\begin{aligned}
\Phi=\left(A \epsilon^{n z}\right. & \left.+B \epsilon^{-\mu z}\right)\left[\sum_{m=0}^{\infty} C_{m} C e_{m}(\xi, s) c e_{m}(\eta, s)\right. \\
& \left.+\sum_{m=1}^{\infty} S_{m} S e_{m}(\xi, s) \operatorname{se}_{m}(\eta, s)\right]
\end{aligned}
$$

where $\Phi$ is the complex effective value of velocity potential which is represented by $\phi=\sqrt{2} \Phi \exp (j \omega t)$. $A, B, C_{m}$ and $S_{m}$ are arbitrary constants determinable from the boundary conditions. Function $c e_{m}(\eta, s), s e_{m}(\eta, s)$ and $C e_{m}(\xi, s), S e_{m}(\xi, s)$ are the Mathieu functions and the modified Mathieu functions of $m$ th-order, respectively.

The value of $s$ is defined by the following equation

$$
s=q^{2}\left(k^{2}+\mu^{2}\right) / 4
$$

where $q$ is the distance between the foci and the origin, $k=\omega / c$ with $\omega$ and $c$ are the angular frequency and the sound velocity, respectively, and $\mu$ is arbitrary constant.

The expansion of Mathieu functions and the 
modified Mathieu functions are defined as follows ${ }^{3)}$

$$
\begin{aligned}
C e_{2 n}(\xi, s)= & c e_{2 n}(j \xi, s)=\sum_{r=0}^{\infty} A_{2 r}^{(2 n)} \cosh 2 r \xi \\
= & \sqrt{\frac{\pi}{2}}(-1)^{n} \sum_{r=0}^{\infty}(-1)^{r} A_{2 r}^{(2 n)} J_{2 r} \\
& \cdot(2 \sqrt{s} \cosh \xi) \\
C e_{2 n+1}(\xi, s)= & c e_{2 n+1}(j \xi, s) \\
= & \sum_{r=0}^{\infty} A_{2 n+1}^{(2 n+1)} \cosh (2 r+1) \xi \\
= & \sqrt{\frac{\pi}{2}}(-1)^{n} \sum_{r=0}^{\infty}(-1)^{r} A_{2 r+1}^{(2 n+1)} J_{2 r+1} \\
& \cdot(2 \sqrt{s} \cosh \xi) \\
S e_{2 n+1}(\xi, s)= & -j s e_{2 n+1}(j \xi, s) \\
= & \sum_{r=0}^{\infty} B_{2 r+1}^{(2 n+1)} \sinh (2 r+1) \xi \\
S e_{2 n+2}(\xi, s)= & -j s e_{2 n+2}(j \xi, s) \\
= & \sum_{r=0}^{\infty} B_{2 r+2}^{(2 n+2)} \sinh (2 r+2) \xi
\end{aligned}
$$

where $J_{m}(x)$ is the Bessel functions of $m$ th-order, and the constants $A_{m}^{(m+\beta)}$ are given as follows

$$
\begin{aligned}
& \sum_{r=0}^{\infty}\left[A_{2 r+1}^{(2 n+1)}\right]^{2}=\sum_{r=0}^{\infty}\left[B_{2 r+1}^{(2 n+1)}\right]^{2}=\sum_{r=0}^{\infty}\left[B_{2 r+2}^{(2 n+2)}\right]^{2}=1 \\
& 2\left[A_{0}^{(2 n)}\right]^{2}=\sum_{r=1}^{\infty}\left[A_{2 r}^{(2 n)}\right]^{2}=1
\end{aligned}
$$

\subsection{Characteristics of Elliptic Cylindrical Cham- bers}

We shall consider the case of elliptic cylindrical chambers of eccentricity $e$ and length $l$, fitted with an elliptical piston of eccentricity $e_{1}$ at the center. The co-ordinates is illustrated in Fig. 1.

Let $V_{\xi}$ and $V_{z}$ be the components of particle velocity which propagates in the $\xi$ and $z$ direction, respectively, and let $V_{0}$ be the driving velocity, the boundary conditions are as follows:
1) $V_{\xi}=-\delta \Phi / \delta \xi=0$
at $\xi=\xi_{0}$
2) $V_{z}=-\delta \Phi / \delta z=0$
at $z=l$
3) $V_{z}=-\delta \Phi / \delta z=F(\xi, \eta) V_{0} \quad$ at $z=0$

where

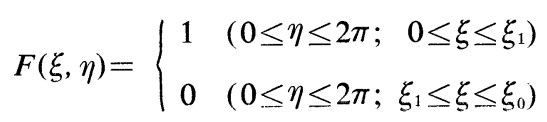

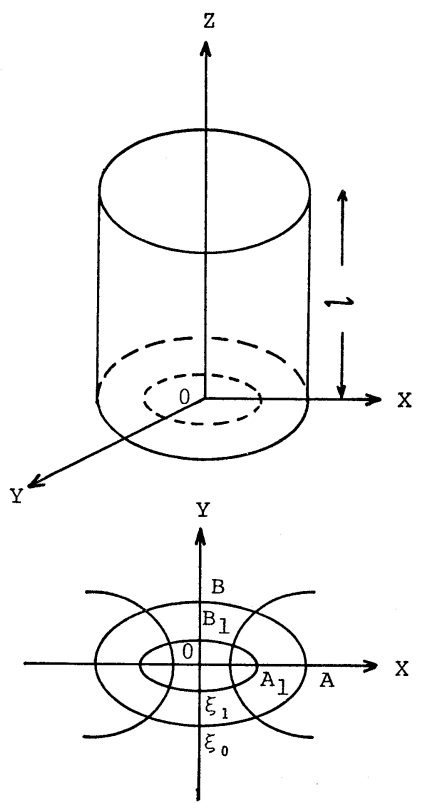

Fig. 1 Elliptic cylindrical chambers and its co-ordinates.

If the distribution of the velocity potential $\Phi$ is symmetrical about the major axis, $\Phi$ must be even and periodic in $\eta$, with period $\pi$ and if it is symmetrical about the minor axis, $\Phi$ must be odd and periodic in $\eta$ with period $2 \pi$. Practically, the input pipe is usually fitted in position on the major axis, hence the solution of Eq. (1) becomes

$$
\Phi=\left(A \epsilon^{\mu z}+B \epsilon^{-\mu z}\right) \sum_{m=0}^{\infty} C_{m} C e_{m}(\xi, s) c e_{m}(\eta, s)
$$

The first boundary condition is satisfied by requiring that $s$ have those values that make

$$
\left.\frac{\delta}{\delta \xi} C e_{m}(\xi, s)\right|_{\xi=\xi_{0}}=0
$$

The positive parametric roots of Eq. (5) are designates as $s_{m, i}$. From the second boundary condition, the relation between $A$ and $B$ is determined as follows:

$$
B=A \epsilon^{2 \mu l}
$$

Thus, Eq. (4) becomes

$$
\begin{aligned}
\Phi=\sum_{m=0}^{\infty} \sum_{i=0}^{\infty} A_{m, i} \frac{2 \cosh \mu_{m, i}(l-z)}{\epsilon^{-\mu_{m, i} l}} \\
\cdot C_{m} C e_{m}\left(\xi, s_{m, i}\right) c e_{m}\left(\eta, s_{m, i}\right)
\end{aligned}
$$




\section{N. M. CUONG and J. OKDA: ELLIPTIC CYLINDRICAL MUFFLER CHAMBERS}

According to the third boundary condition, we have

$$
\begin{aligned}
-\left.\frac{\delta \Phi}{\delta z}\right|_{z=0}=V_{0} F(\xi, \eta)=\sum_{m=0}^{\infty} \sum_{i=0}^{\infty} A_{m, i} \\
\quad \cdot \frac{2 \mu_{m, i} \sinh \mu_{m, i} l}{\boldsymbol{\epsilon}^{-\mu_{m, i} l}} \operatorname{Cem}\left(\xi, s_{m, i}\right) \operatorname{ce}\left(\eta, s_{m, i}\right)
\end{aligned}
$$

By multiplying both side of Eq. (7) by $\mathrm{Ce}\left(\xi, s_{m, i}\right)$ $c e\left(\eta, s_{m, i}\right)(\cosh 2 \xi-\cos 2 \eta)$ and integrating with respect to $\eta$ from 0 to $2 \pi$, and with respect to $\xi$ from 0 to $\xi_{0}$, the constant $A_{m, i}$ is determined as follows:

$$
\begin{aligned}
& A_{m, i}= \\
& V_{0} \frac{\epsilon^{-\mu_{m, i} l} \int_{0}^{\xi_{1}} C e_{m}\left(\xi, s_{m, i}\right)\left[2 A_{m} \cosh 2 \xi-A_{m}^{\prime}\right] d \xi}{2 \mu_{m, i} \sinh \mu_{m, i} l \int_{0}^{\xi_{0}} C e_{m}^{2}\left(\xi, s_{m, i}\right)\left[\cosh 2 \xi-\theta_{m, i}\right] d \xi}
\end{aligned}
$$

where

$$
\begin{aligned}
& A_{m}=A_{0}^{(2 n)} ; \quad A_{m}^{\prime}=A_{2}^{(2 n)} ; \\
& \theta_{m, i}=A_{0}^{(2 n)} A_{2}^{(2 n)}+\sum_{r=0}^{\infty} A_{2 r}^{(2 n)} A_{2 r+2}^{(2 n+1)} \\
& \quad m=2 n(n=0,1,2, \ldots) \\
& A_{m}=A_{1}^{(2 n+1)} ; \quad A_{m}^{\prime}=0 ; \\
& \theta_{m, i}=\frac{1}{2}\left[A_{1}^{(2 n+1)}\right]^{2}+\sum_{r=0}^{\infty} A_{2 r+1}^{(2 n+1)} A_{2 r+3}^{(2 n+1)} \\
& \quad m=2 n(n=0,1,2, \ldots)
\end{aligned}
$$

Substituting Eq. (8) into Eq. (6) and expanding it with mode $(0,0)$ leads to

$$
\begin{aligned}
\Phi=- & V_{0}\left[\frac{S_{1}}{S}\left(\frac{A^{2}-B^{2}}{A_{1}^{2}-B_{1}^{2}}\right) \frac{\cos (l-z)}{k \sin k l}\right. \\
& -\sum_{m=0}^{\infty} \sum_{i=1}^{\infty} \frac{\cosh \mu_{m, i}(l-z)}{\mu_{m, i} \sinh \mu_{m, i} l} \\
& \cdot C e_{m}\left(\xi, s_{m, i}\right) c e_{m}\left(\eta, s_{m, i}\right) \\
& \left.\cdot \frac{\int_{0}^{\xi_{1}} C e_{m}\left(\xi, s_{m, i}\right)\left[2 A_{m} \cosh 2 \xi-A_{m}^{\prime}\right] d \xi}{\int_{0}^{\xi_{0}} C e_{m}^{2}\left(\xi, s_{m, i}\right)\left[\cosh 2 \xi-\theta_{m, i}\right] d \xi}\right]
\end{aligned}
$$

where $A, B, A_{1}$ and $B_{1}$ are the half length of the major and minor axes of the chamber and the input pipe, respectively, and $S$ and $S_{1}$ are these crosssectional areas. The sound pressure on the output side plate $(z=l)$ is given by the following equation

$$
\begin{aligned}
P= & j k \rho c(\Phi)_{z=l} \\
= & -j Z U_{0}\left[\left(\frac{A^{2}-B^{2}}{A_{1}^{2}-B_{1}^{2}}\right) \frac{1}{\sin k l}-\sum_{m=0}^{\infty} \sum_{i=1}^{\infty} \frac{S}{S_{1}} \frac{k}{\mu_{m, i}}\right. \\
& \cdot \frac{1}{\sinh \mu_{m, i} l} C e_{m}\left(\xi, s_{m, i}\right) c e_{m}\left(\eta, s_{m, i}\right) \\
& \left.\cdot \frac{\int_{0}^{\xi_{1}} C e_{m}\left(\xi, s_{m, i}\right)\left[2 A_{m} \cosh 2 \xi-A_{m}^{\prime}\right] d \xi}{\int_{0}^{\xi_{0}} C e_{m}^{2}\left(\xi, s_{m, i}\right)\left[\cosh 2 \xi-\theta_{m, i}\right] d \xi}\right]
\end{aligned}
$$

where $Z=\rho c / S$ is the characteristic impedance of the chamber and $U_{0}=V_{0} S_{1}$ is the volume velocity supplied from the input pipe. The characteristics of an expansion chamber as an element of a muffler system is represented by $20 \log \left|Y_{f} Z_{3}\right|$, where $Y_{f}=$ $U_{0} / P$ is the transfer admittance of the chamber and $Z_{3}$ is the characteristic impedance of the output pipe. ${ }^{1)}$

\subsection{Resonance Frequencies}

The first term of Eq. (10) represents the sound pressure component of plane wave and the second term represents the sound pressure components of traverse waves. These resonances are in the frequencies at which the denominators of Eq. (10) are zero, namely

$$
\left.\begin{array}{c}
\sin k l=0, \quad k A=\alpha \pi A / l \\
(\alpha=0,1,2, \ldots)
\end{array}\right\}
$$

and

$$
\left.\begin{array}{l}
\sinh \mu_{m, i} l=0 \\
k A=\sqrt{\left(\frac{2 \sqrt{s_{m, i}}}{e}\right)^{2}+\left(\frac{2 \alpha \pi A}{l}\right)^{2}}
\end{array}\right\}
$$

In order to define the values $k A$ at the fundamental resonance of each mode, first giving $s$ of Eq. (3a) and Eq. (3b) suitable values corresponding to each mode, and the value of $\xi_{0}$ is determined so as to satisfy Eq. (5). With obtained $\xi_{0}$ by using the relation $e=1 / \cosh \xi_{0}$, we can find $k A$ as follows ${ }^{4}$ )

$$
k A=2 \sqrt{s} \cosh \xi_{0}
$$

where the constant $A_{m}^{(m+\beta)}$ in Eq. (3a) and Eq. (3b) are referred to the tables relating to the Mathieu functions. ${ }^{5)}$

Relationship between eccentricity $e$ and $k A$ which 


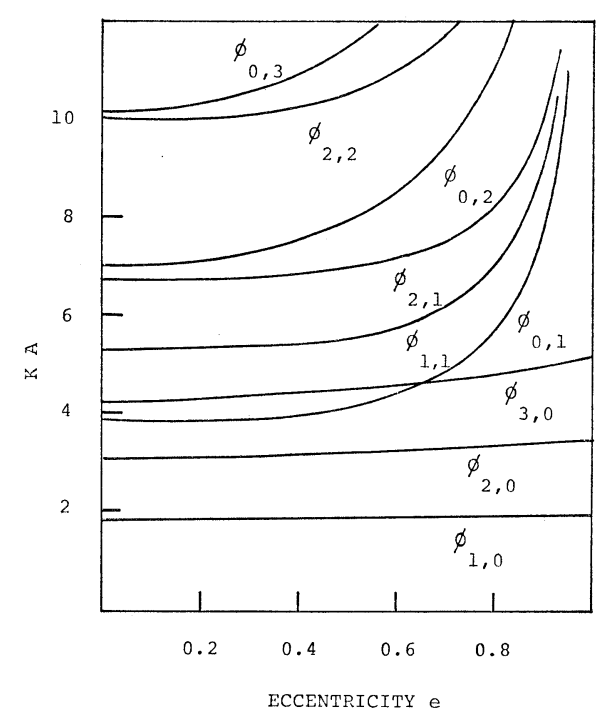

Fig. 2 Fundamental resonances of even waves.

corresponds to the higher modes is illustrated in Fig. 2.

\section{DISCUSSION}

As mentioned in section 2.3, the second term of Eq. (10) represents the sound pressure components of traverse waves. In the range where higher modes are generated, the value of $\left|Y_{f}\right|$ does not decrease as much as in the case of circular chambers. However, the sound pressure will increase near the resonance frequencies and when the resonance frequencies of other modes co-occur, $\left|Y_{f}\right|$ will be small and the noise reduction can not be expected to be as great.

Hereafter, in order to obtain a wide effective frequency range for the chamber of a muffler, we will investigate the characteristics of the higher modes and define the position of the input and output pipes.

At first, when the function $\mathrm{Ce}\left(\xi, s_{m, i}\right)$ or $c e(\eta$, $\left.s_{m, i}\right)$, the second term of Eq. (10), is zero, the sound pressure of $(m, i)$ mode will be eliminated. The former represents the confocal nodal ellipse and the latter represents the confocal nodal hyperbola of that mode. Shapes of the first 4 normal modes are shown in Fig. 3.

In Fig. 3, the shapes of the sound pressure distribution of odd and even waves in both circle and

\begin{tabular}{|c|c|c|c|c|}
\hline \multirow{2}{*}{ Mode } & \multicolumn{2}{|c|}{ Circle } & \multicolumn{2}{c|}{ Ellipse } \\
\cline { 2 - 5 } & even & odd & even & odd \\
\hline$(0,0)$ & & & & \\
\hline$(1,0)$ & & & & \\
\hline$(2,0)$ & & & & \\
\hline$(0,1)$ & 0 & & & \\
\hline$(3,0)$ & & & & \\
\hline
\end{tabular}

Fig. 3 Shapes of some of the normal modes of circle and ellipse.

ellipse are shown together. The dark and the light area represent positive and negative sound pressure, respectively, and the boundary represents the confocal nodal hyperbola or ellipse of that mode. As illustrated in Fig. 3, when an output or an input pipe is not fitted on the major or minor axes, the second term of Eq. (1) does not vanish hence the resonance of even and odd waves will occur in the chamber. On the other hand, when they are fitted on the major axis, odd wave components disappear and only the resonance of even waves are generated in the chamber. In case the input or output pipe is fitted at the center, the $(1,0)$ mode will be eliminated.

Figure 4 indicates the calculated value of the ratio $P / P_{1}$ in $\mathrm{dB}$ when the input and output pipes are fitted at the center of the chamber $(e=0.8, A=$ $160 \mathrm{~mm}, l=200 \mathrm{~mm})$. Where $P_{1}$ and $P$ are the sound pressure at input and output pipes, respectively. The relation between $\left|P / P_{1}\right|$ and $\left|Y_{j}\right|=\left|U_{0} / P\right|$ is shown by Eq. (16) later and the sound pressure of each mode is calculated by Eqs. (10) and (16). Let $\lambda_{m, i}$ be the value of $k A$ where the fundamental resonance of mode $(m, i)$ is generated. $\left|P / P_{1}\right|$ of the mode becomes infinite at $k A=\lambda_{m, i}$ and it decreases in proportion to $\left|k / \mu_{m, i} \sin \mu_{m, i} l\right|$ in terms of the increases of $k A$. On the other hand, at $k A>\lambda_{m, i}$, $\left|P / P_{1}\right|$ of the mode is small in proportion to $\mid k / \mu_{m}, i$ $\sinh \mu_{m, i} l \mid$ and this mode can be ignored. Therefore, it is not necessary to consider the higher modes if $A$ is chosen to satisfy the condition of $k A<\lambda_{2,0}$ 


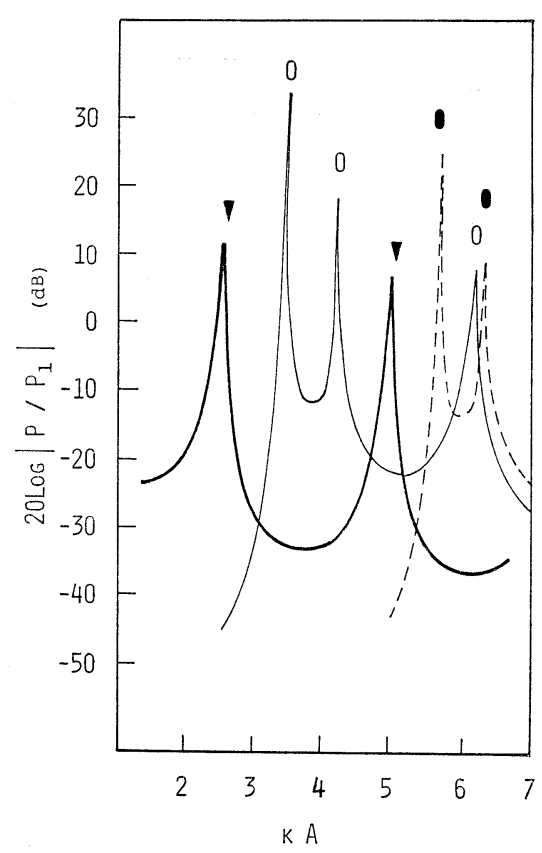

Fig. 4 Calculated results by Eqs. (10) and (16) $(e=0.8, A=160 \mathrm{~mm}, l=200 \mathrm{~mm})$. $\nabla$; resonance of $(0,0)$ mode, $\bigcirc$; resonance of $(2,0)$ mode, $\bullet$; resonance of $(0,1)$ mode.

by designing the elliptical chamber, and in this case, $\left|Y_{f}\right|$ is expressed by the following equation

$$
Y_{f}=\frac{U_{0}}{P}=j \frac{1}{Z} \frac{\left(A_{1}^{2}-B_{1}^{2}\right)}{\left(A^{2}-B^{2}\right)} \sin k l
$$

From the comparison between the effective frequency range of the circular chamber and that of the elliptical chamber, the following discussion will hold good.

If both of the input and output pipes are fitted at the center of the cross section, the effective frequency range of elliptical chamber will be narrower than that of the circular chamber which has a nodal line of $(2,0)$ mode on the central position as illustrated in Fig. 3. Therefore, it is advisable that one of the input or output pipes is fitted at the center and the other is fitted at the intersection of confocal nodal hyperbola of $(2,0)$ mode and the major axis. In this case the effect of $(2,0)$ mode will be eliminated and the effective frequency range will be spread to a point of $k A<\lambda_{0,1}$ as in the case

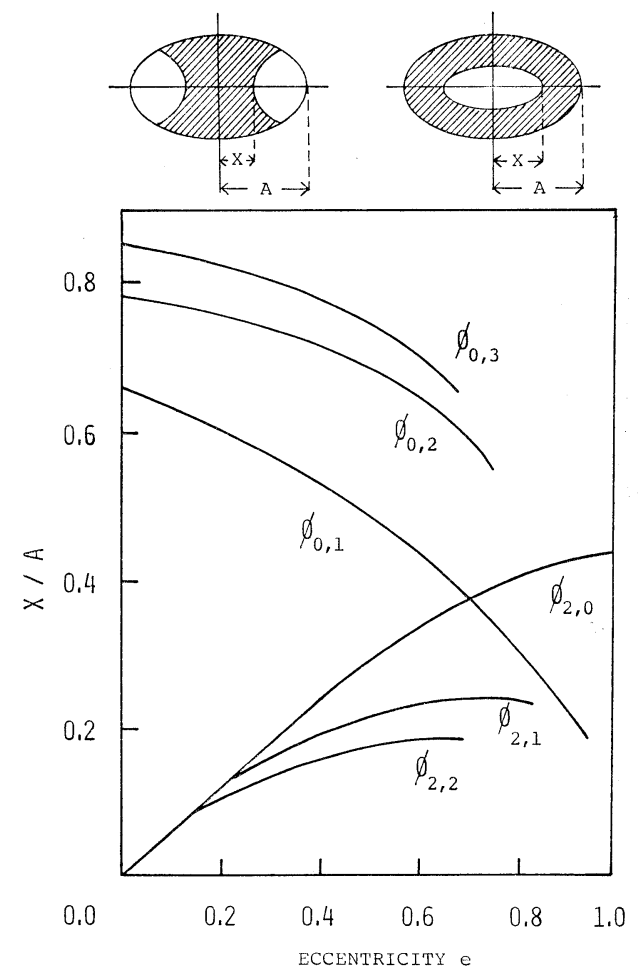

Fig. 5 Position of some confocal nodal hyperbola, ellipse on major axis.

of the circular chamber. Additionally, if we choose a high value of $e$, with the same order normal mode, the resonance frequency of the elliptical chamber will be higher than that of the circular chamber as shown in Fig. 2, namely the effective frequency range of the former will become wider.

The relationship between eccentricity $e$ and $X / A$, is shown in Fig. 5, where $X$ is the distance from the origin to the confocal nodal ellipse or hyperbola along major axis and $A$ is the half length of the major axis. These curves are calculated from Eq. (5) and the following equation

$$
\begin{aligned}
& \left.C e_{m}(\xi, s)\right|_{s=s_{m}, i}=0 ;\left.\quad c e_{m}(\eta, s)\right|_{s=s_{m}, i}=0 ; \\
& X / A=e \cosh \xi \cos \eta
\end{aligned}
$$

Note that if we chose $e=0.67$, and if one of the input or output pipes is fitted at the center and the other at $X=0.37 \mathrm{~A}$, it is probable that the effects of $(2,0)$ and $(0,1)$ modes will be eliminated. In 


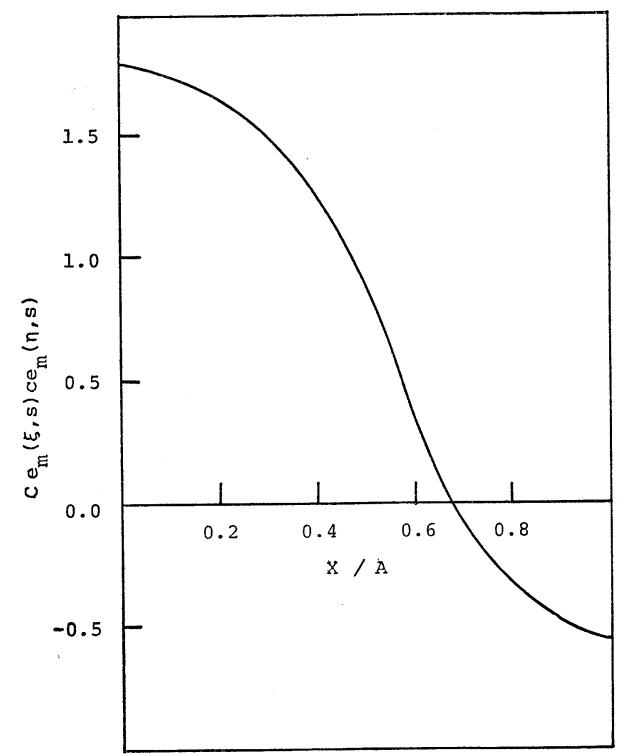

Fig. 6 Curve of $(0,1)$ mode.

practice, the component of $(0,1)$ mode will not be eliminated completely because the cross sectional area of the input and output pipes are not small enough and the sound pressure of $(0,1)$ mode distributes nonsymmetrically as shown in Fig. 6.

\section{EXPERIMENTS}

A block diagram of the whole experimental apparatus is shown in Fig. 7. In order to maintain the sound pressure $\boldsymbol{P}_{1}$ constantly, the output of microphone (1) fitted at the input pipe was transfered to the compressor circuit, and the sound pressure $P$ is recorded by a level recorder which was driven by an oscillator. The relationship between $|P|$ and $\left|P_{1}\right|$ is described by the following equation

$$
\begin{aligned}
20 \log \left|\frac{\boldsymbol{P}}{\boldsymbol{P}_{1}}\right|= & -20 \log \left|\frac{U_{0}}{\boldsymbol{P}}\right| \\
& -20 \log \left|Z_{0} \sin k L_{0}^{\prime}\right|
\end{aligned}
$$

where $Z=\rho c / S$ is the characteristic impedance and $L_{0}{ }^{\prime}=L_{0}+0.8 A_{0}$ is the effective length of the input pipe.

The chamber being made of an iron plate has eccentricity $e=0.8 \quad(A=160 \mathrm{~mm})$ and length $l=$ $200 \mathrm{~mm}$. The circular input pipe with a diameter of $33 \mathrm{~mm}$ and length $50 \mathrm{~mm}$ is used instead of the elliptical pipe. The results are shown in Figs.

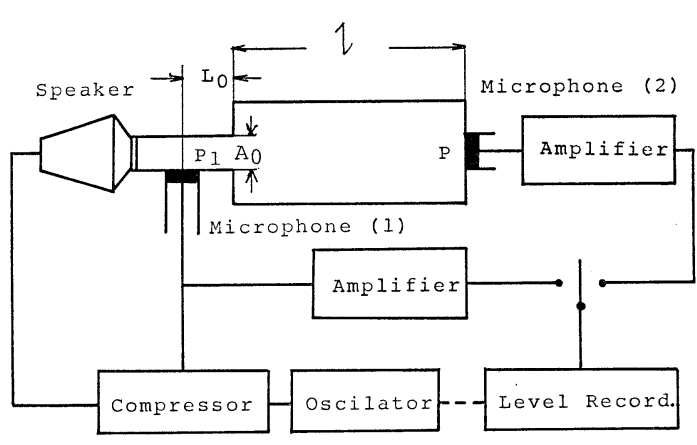

Fig. 7 Block diagram of the experimental apparatus $(e=0.8, A=160 \mathrm{~mm}$, $\left.l=200 \mathrm{~mm}, L_{0}{ }^{\prime}=30 \mathrm{~mm}, A_{0}=30 \mathrm{~mm}\right)$.
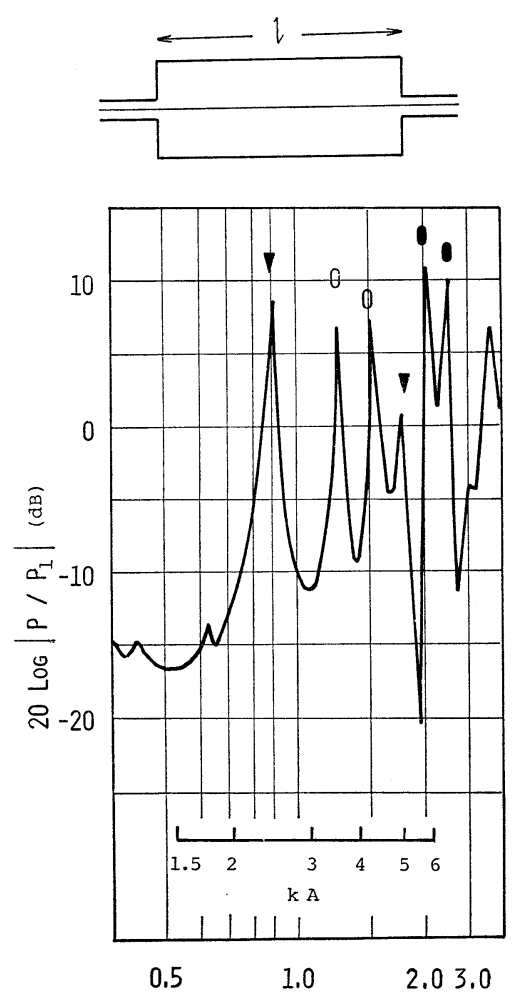

FREQUENCY ( $\mathrm{KHz})$

Fig. 8 Characteristics of elliptic cylindrical chamber ( $e=0.8, A=160 \mathrm{~mm}, l=200 \mathrm{~mm}$ ). $\mathbf{\nabla}$; resonance of $(0,0)$ mode, $O$; resonance of $(2,0)$ mode, $\bullet$; resonance of $(0,1)$ mode. 

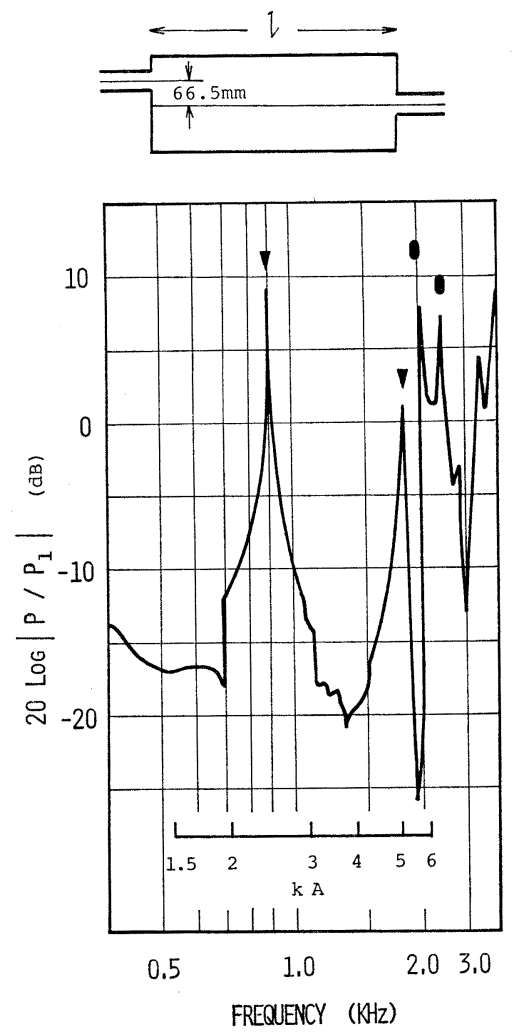

Fig. 9 Characteristics of elliptic cylindrical chamber $(e=0.8, A=160 \mathrm{~mm}, l=200 \mathrm{~mm})$. $\mathbf{\nabla}$; resonance of $(0,0)$ mode, $\bullet$; resonance of $(0,1)$ mode.

$8 \sim 10$.

Figure 8 shows the characteristics of the chamber. In the case both the input and the output pipes are fitted at the center. Except for a difference in the whole level caused by the loss in the chamber and the loss in the input pipe, the resonance frequencies and the general characteristics agree well with the theoretical results shown in Fig. 4. In Fig. 9 the output pipe is fitted at the center and the input pipe is fitted at the intersection of the confocal nodal hyperbola of $(2,0)$ mode and the major axis. The sound pressure component of $(2,0)$ mode is completely eliminated by its symmetrical distribution through the major axis. In this case the upper limit of the effective frequency range is given in the area below the fundamental resonance frequency of $(0,1)$ mode. Note that the resonance of $(0,1)$ mode will occur near $1.4 \mathrm{kHz}$ in a circular
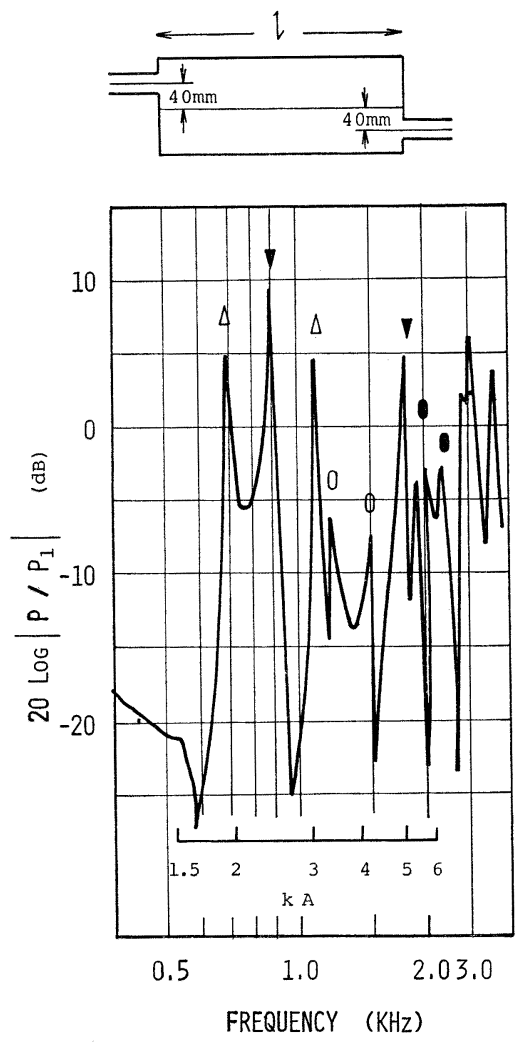

Fig. 10 Characteristics of elliptic cylindrical chamber $(e=0.8, \quad A=160 \mathrm{~mm}, \quad l=200$ $\mathrm{mm}$ ).

$\mathbf{\nabla}$; resonance of $(0,0)$ mode, $\Delta$; resonance of $(1,0)$ mode, $O$; resonance of $(2,0)$ mode, $\bullet$; resonance of $(0,1)$ mode.

chamber of the same cross-sectional area.

In Fig. 10 the input and output pipes are fitted at the positions on the major axis different from the cases of Fig. 8 and Fig. 9. As mentioned above the resonance of all higher modes which have both odd and even waves will increase the whole level. Furthermore the effective frequency range will become narrower.

\section{CONCLUSION}

In order to make clear the characteristics of elliptic cylindrical chambers of a muffler, the distribution of sound pressure is calculated by solving the wave equations, assumming that the loss can be neglected. In addition, the effective frequency range is defined by theoretical investigation and 
verified by experiment on the formation of higher modes and on the position of input and output pipes.

The results obtained show that theoretical values related to the distribution of sound pressure inside the chambers and resonance frequencies agree well with the experimental values. Also when one of the input or output pipes is fitted at the center and the other at the confocal nodal hyperbola of $(2,0)$ mode on the major axis, the effective frequency range of the elliptical chamber will be wider than that of the circular chamber.

\section{REFERENCES}

1) J. Okda, "Characteristics of the cylindrical cham- ber of the expansion chamber type muffler," J. Acoust. Soc. Jpn. 29, 199-206, (1973) (in Japanese).

2) J. Okda, "Four-terminal constants of co-axial cylindrical cavities," J. Acoust. Soc. Jpn. 31, 608613, (1975) (in Japanese).

3) N. W. McLachlan, Theory and Applications of Mathieu Function (Clarendon Press, Oxford, 1951), 267-330.

4) A. Hasegawa, "Comparisons of sound propagation in rigid walled and soft-walled elliptical waveguides,” J. Acoust. Soc. Jpn. 32, 703-709, (1976) (in Japanese).

5) National Bureau of Standards, Tables Relating to Mathieu Function (Columbia Univ. Press, New York, 1951), 41-150. 